\title{
PHASE PORTRAITS OF UNIFORM ISOCHRONOUS CENTERS WITH HOMOGENEOUS NONLINEARITIES
}

\author{
JAUME LLIBRE $^{1}$ AND CLAUDIA VALLS ${ }^{2}$
}

\begin{abstract}
We classify the phase portraits in the Poincare disc of the differential equations of the form $x^{\prime}=-y+x f(x, y), \dot{y}=x+y f(x, y)$ where $f(x, y)$ is a homogeneous polynomial of degree $n-1$, and $f$ has only simple zeroes when $n=2,3,4,5$. We also provide some general results on these uniform isochronous centers for all $n \geq 2$.
\end{abstract}

\section{INTRODUCTION AND STATEMENT OF THE MAIN RESULTS}

The first investigation in isochronicity goes back to Huygens in [6] with the study of the cycloidal pendulum in the XVII century. Nowadays isochronicity appears in many physical problems and it is closely related with the existence and uniqueness of solutions for certain bifurcation problems or boundary value problems (see for instance [8] and the references therein). In the last decade the study of the isochronicity has been grown specially in the case of polynomial differential systems due to the appearance of powerful methods of computational analysis, see for instance $[1,3,4,14]$ to cite just few of them.

A polynomial differential system of degree $n$ is a differential system

$$
\dot{x}=P(x, y), \quad \dot{y}=Q(x, y),
$$

with $P$ and $Q$ polynomials such that the maximum of their degrees is $n$. We denote by $\chi=(P, Q)$ the polynomial vector field associated to system (1).

Let $p \in \mathbb{R}^{2}$ be a center of $\chi$. We say that $p$ is an isochronous center of $\chi$ if it is a center with a neighborhood surrounded by periodic orbits with the same period. We say that $p$ is a uniform isochronous center of $\chi$ if the system associated to $\chi$ can be written in polar coordinates in the form

$$
\dot{r}=G(\theta, r), \quad \dot{\theta}=\kappa, \quad \kappa \in \mathbb{R} \backslash\{0\} .
$$

We say that $p$ is a global center if its period annulus is $\mathbb{R}^{2}$. We recall that a period annulus of a center $p$ is the maximum neighborhood $U \subset\{p\}$ of the center $p$ filled up with periodic orbits.

2010 Mathematics Subject Classification. Primary 34A05. Secondary 34C05, 37C10. Key words and phrases. Polynomial vector field, uniform isochronous center, phase portrait, Poincaré disc. 
The polynomial differential systems can be extended analytically to infinity in the so called Poincaré compactification. Roughly speaking, the Poincare compactification identify $\mathbb{R}^{2}$ with the interior of the unit disc centered at the origin and its boundary, the circle $\mathbb{S}^{1}$, with the infinity of $\mathbb{R}^{2}$, in the plane we can go to infinity in as many directions as points have $\mathbb{S}^{1}$. Then the polynomial differential system can be extended to an analytic differential system in the closed unit disc, i.e. in particular to the infinity its infinity $\mathbb{S}^{1}$. This closed disc is called the Poincare disc. The equilibrium points of the extended differential system in $\mathbb{S}^{1}$ are called the infinite equilibria of the initial polynomial differential system. For more details on this Poincaré compactification see Chapter 5 of [5].

Two polynomial vector fields are topologically equivalent if there exists a homeomorphism which preserves the infinity carrying orbits of the flow induced by the first compactified vector field to orbits of the flow induced by the second compactified vector field.

To obtain the phase portrait of a polynomial vector field with finitely many separatrices, we need to determine the separatrices of the compactified vector field and one orbit inside each canonical region, for more details see Chapter 1 of [5].

A limit cycle is a periodic orbit of system (1) isolated in the set of all periodic orbits of system (1).

A non locally constant $C^{1}$ function $H: U \rightarrow \mathbb{R}^{2}$ (where $U$ is an open and dense subset of $\mathbb{R}^{2}$ ) is a first integral of system (1) in $U$ if $H$ is constant on the solution curves of system (1), or equivalently

$$
P(x, y) H_{x}+Q(x, y) H_{y}=0,
$$

in $U$. Of course, $H_{x}$ denotes the partial derivative of $H$ with respect to $x$. System (1) has a Liouvillian first integral if it has an integrating factor given by a Darboux function (see section 2 for the definition for integrating factor and Darboux function).

In the present paper we will classify the phase portraits in the Poincaré disc of uniform isochronous centers of the polynomial differential system

$$
\dot{x}=-y+x f(x, y), \quad \dot{y}=x+y f(x, y),
$$

with homogeneous polynomial nonlinearities $x f(x, y)$ and $y f(x, y)$, being $f(x, y)$ a homogenous polynomial of degree $n-1$.

The line at infinity of the polynomial differential systems (1) is filled up with singularities. When we remove this line of singularities, it can remain some additional singularities, that we call special singularities.

The following is our first main result.

Theorem 1. Consider the polynomial differential system (2) of degree $n \geq 2$ such that $f$ is a homogeneous polynomial of degree $n-1$ that we shall write 

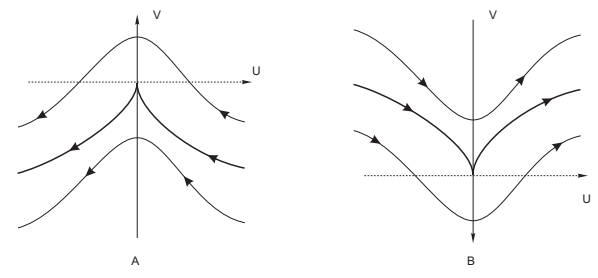

Figure 1. The cups at infinity.

$a s$

$$
f(x, y)=\sum_{j=0}^{n-1} f_{j} x^{n-1-j} y^{j} .
$$

The following holds.

(a) The unique finite singular point is the origin.

(b) It has a Liouvillian first integral.

(c) If $n$ is even, the origin is a uniform isochronous center, and if $n$ is odd and

$$
\sum_{j=0}^{n-1} f_{j} \int_{0}^{2 \pi} \cos ^{n-1-j} \theta \sin ^{j} \theta=0,
$$

then it is a uniform isochronous center, otherwise it is a focus.

(d) In case that the origin is a focus, the system cannot have limit cycles.

(e) If $n>1$ and the origin is a center it cannot be a global center.

(f) The line at infinity is filled up with singularities.

(g) If the origin is a center, then there is at least one special singularity except if $f(x, y)=x^{n-1}$.

(h) Assume that all $k \leq n-1$ real zeros of the polynomial $f(1, y)$ are simple. Then the following statements hold.

(h.1) If $n$ is even all the special singular points at infinity are cusps and their local phase portrait might be one of the two shown in Figure 1. Moreover between two cusps with local phase portrait as in Figure 1.A there must be a cusp with local phase portrait as in Figure 1.B.

(h.2) If $n$ is odd then $k \geq 2$ is even and there are $k / 2$ special singularities at infinity which are saddles and $k / 2$ special singularities at infinity which are centers or foci. Moreover between two saddles there must be a center or a focus.

The proof of statement (e) is Theorem 3.1 of [4] but the proof there is more complicated than the one done in this paper and so we decided to include it here. Theorem 1 is proved in section 3 .

Theorem 2. Consider the polynomial differential system (2) of degree $n \geq 2$ such that $f$ is a homogeneous polynomial of degree $n-1$ satisfying (3) when 


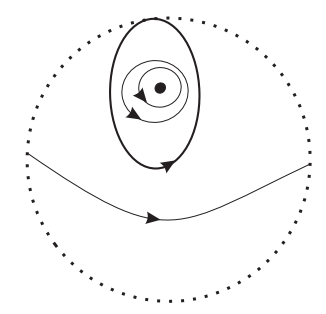

Figure 2. Phase portraits of the polynomial differential system (2) with $n=2$.

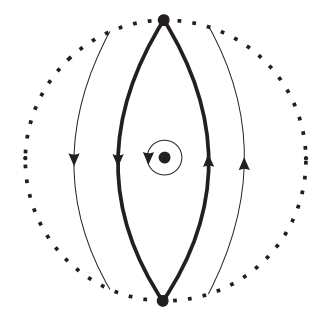

Figure 3. Phase portraits of the polynomial differential system (2) with $n=3$.

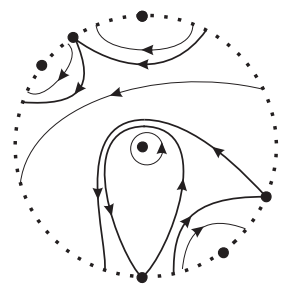

A
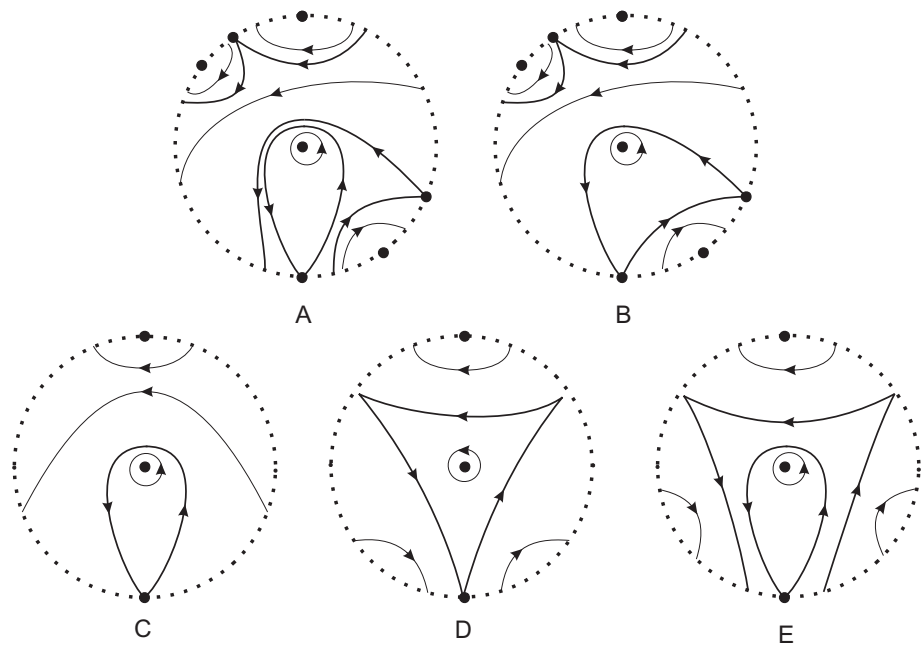

Figure 4. Phase portraits of the polynomial differential system (2) with $n=4$.

$n$ is odd. The phase portraits in the Poincaré disc for $n=2,3,4$ are given in Figures 2, 3 and 4, respectively. Moreover, Figure 2 is achieved with the 
polynomial differential system

$$
\dot{x}=-y+x^{2}, \quad \dot{y}=x(1+y) .
$$

Figure 3 is achieved with the polynomial differential system

$$
\dot{x}=-y+x^{2} y, \quad \dot{y}=x+x y^{2},
$$

and the local phase portraits (a), (b), (c), (d) and (e) of Figure 4 are achieved with the polynomial differential systems

$$
\dot{x}=-y-x^{2}(x+a y)(x+2 y), \quad \dot{y}=x-x y(x+a y)(x+2 y),
$$

with $a=1$,

$$
\dot{x}=-y-x^{2}(x+a y)(x+2 y), \quad \dot{y}=x-x y(x+a y)(x+2 y),
$$

with $a \in(9 / 16,5 / 8)$,

$$
\begin{array}{ll}
\dot{x}=-y-x y\left(y^{2}-3 x^{2}\right), & \dot{y}=x-y^{2}\left(y^{2}-3 x^{2}\right), \\
\dot{x}=-y+x^{2}\left(5 y^{2}-x^{2}\right), & \dot{y}=x-x y\left(5 y^{2}-x^{2}\right),
\end{array}
$$

and

$$
\dot{x}=-y-x^{2}\left(x^{2}+y^{2}\right), \quad \dot{y}=x-x y\left(x^{2}+y^{2}\right),
$$

respectively.

The proof of Theorem 2 for the case $n=2$ is given in [13]. We recall that there is no infinite special singularity in this case.

The proof of Theorem 2 for the case $n=3$ is given in [2]. We recall that there are two special singularities that are a saddle and a center or focus.

The proof of Theorem 2 for the case $n=4$ is given in [12]. We recall that in the phase portraits A, B, C and D of Figure 4 there are three special singularities that are cusps and in Figure 4.E there is only one special singularity (again a cusp). Figure 4.E can also be realized without any special singularity for example in system

$$
\dot{x}=-y+x^{4}, \quad \dot{y}=x+y x^{3} .
$$

Figures 4.C and 4.D are missing in [12]. Here we provide the complete characterization for systems (2) with homogeneous nonlinearities of degree $n=4$.

The following is the second main theorem of the paper.

Theorem 3. Consider the polynomial differential system (2) of degree $n=5$ such that $f$ is a homogenous polynomial of degree 4 satisfying (3) and with all its real zeros being simple. The phase portraits in the Poincaré disc are given in Figure 3 and in Figure 5.A and 5.B. Figure 3 is achieved with the polynomial differential system

$$
\dot{x}=-y-x^{2} y\left(x^{2}+y^{2}\right), \quad \dot{y}=x+x y^{2}\left(x^{2}+y^{2}\right) .
$$



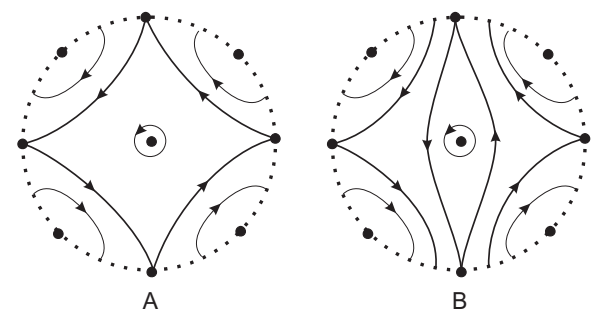

Figure 5. Phase portraits of the polynomial differential system (2) with $n=5$.

Figures 5.A and 5.B are achieved with the polynomial differential systems

$$
\begin{aligned}
& \dot{x}=-y-x^{2}(y-x)(y-2 x)(y+3 x / 7), \\
& \dot{y}=x-x y(y-x)(y-2 x)(y+3 x / 7),
\end{aligned}
$$

and

$$
\dot{x}=-y-x^{2} y\left(y^{2}-x^{2}\right), \quad \dot{y}=x-x y^{2}\left(y^{2}-x^{2}\right),
$$

respectively.

The proof of Theorem 3 is given in section 4 .

\section{Preliminary Results}

In this section we introduce some preliminary results. The proof of the first result can be obtained in [4].

Proposition 4. Assume that the planar polynomial differential system

$$
\dot{x}=P(x, y), \quad \dot{y}=Q(x, y)
$$

of degree $n$ has a center at the origin. Them this center is a isochronous center if and only if by a linear change of variables and a rescaling of time it can be written as in (2), with $f(x, y)$ a polynomial of degree $n-1$ and $f(0,0)=0$.

In the case of isochronous centers with homogeneous nonlinearities the following result was proved in [4] (see Theorem 2.1).

Theorem 5. Let $f$ be a homogeneous polynomial of degree $n-1$ as in the statement of Theorem 1. Then system (2) has a uniform isochronous center at the origin if either $n$ is even or $n$ is odd and condition (3) holds.

The following proposition deals with the existence of limit cycles. Its proof can be found in [7]. We recall that $V$ is an inverse integrating factor 
of a vector field $\chi=(P, Q)$ with $P, Q \in \mathbb{R}[x, y]$ if it satisfies the partial differential equation

$$
P \frac{\partial V}{\partial x}+Q \frac{\partial V}{\partial y}=\left(\frac{\partial P}{\partial x}+\frac{\partial Q}{\partial y}\right) V
$$

Theorem 6. Let $\chi=(P, Q)$ be a $C^{1}$ vector field defined in a subset $U \subset \mathbb{R}^{2}$. Let $V$ be an inverse integrating factor of the vector field. If $\gamma$ is a limit cycle of $\chi$ then $\gamma$ is contained $\Sigma=\{(x, y) \in U: V(x, y)=0\}$.

Let $\mathbb{R}[x, y]$ be the ring of all real polynomials in the variables $x$ and $y$.

An invariant algebraic curve of system (1) is a curve $g(x, y)=0$ where $g \in \mathbb{R}[x, y]$ satisfies

$$
P(x, y) \frac{\partial g}{\partial x}+Q(x, y) \frac{\partial g}{\partial y}=K(x, y) g
$$

where $K \in \mathbb{R}[x, y]$ is called the cofactor of $g$. Note that the curve is invariant by the dynamics of system (1) in the sense that if a trajectory starts on the curve it does not leave it.

An exponential factor of system (1) is a function $F=\exp (g / h)$ with coprime $g, h \in \mathbb{R}[x, y]$ such that

$$
P(x, y) \frac{\partial F}{\partial x}+Q(x, y) \frac{\partial F}{\partial y}=L(x, y) F,
$$

where the polynomial $L \in \mathbb{R}[x, y]$ whose degree is the degree of the system minus one, is called the cofactor of $F$.

We recall that in view of Theorem 8.7 (iv)) of [5] we have that

Proposition 7. Assume that a polynomial differential system $\chi$ admits $p$ invariant algebraic curves $f_{i}=0$ with cofactors $K_{i}$ for $i=1, \ldots, p$ and $q$ exponential factors $F_{i}$ with cofactors $L_{j}$ for $j=1, \ldots, q$. Then there exits $\lambda_{j}, \mu_{j} \in \mathbb{C}$ not all zero such that

$$
\sum_{i=1}^{p} \lambda_{i} K_{i}+\sum_{j=1}^{q} \mu_{j} L_{j}=-\operatorname{div}(\chi)
$$

if and only if the function

$$
f_{1}^{\lambda_{1}} \cdots f_{p}^{\lambda_{p}} F_{1}^{\mu_{1}} \cdots F_{q}^{\mu_{q}}
$$

is an integrating factor of $\chi$. Here div stands for the divergence of the system.

A function of the form in (4) is called a Darboux function.

The following theorem was proved in [15].

Theorem 8. The polynomial differential system (1) has a Liouvillian first integral if and only if it has an integrating factor which is a Darboux function. 
The next result is proved in Theorem 3.5 of [5].

Theorem 9. Let $(0,0)$ be an isolated singular point of system

$$
\dot{x}=y+A(x, y), \quad \dot{y}=B(x, y),
$$

where $A$ and $B$ are analytic in a neighborhood of the point $(0,0)$ and also the first derivatives of $A(x, y)$ and $B(x, y)$ in the variables $x, y$ evaluated at $(0,0)$ are zero. Let $y=f(x)$ be the solution of the equation $y+A(x, y)=0$ in a neighborhood of the point $(0,0)$ and consider $F(x)=B(x, f(x))$ and $G(x)=(\partial A / \partial x+\partial B / \partial y)(x, f(x))$. If $A(x) \not \equiv 0$ and $B(x) \not \equiv 0$ write $F(x)=$ $a x^{m}+$ h.o.t and $G(x)=b x^{n}+$ h.o.t with $m \in \mathbb{N}$ and $m \geq 2, n \in \mathbb{N}, n \geq 1$, $a b \neq 0$. The following holds.

(a) if $m$ is even and $m<2 n+1$, then the origin of $X$ is a cusp.

(b) if $m$ is odd and $a>0$ then the origin of $X$ is a saddle.

(c) if $m$ is odd, $a<0$ and $m<2 n+1$, then the origin of $X$ is a center or a focus.

\section{Proof of Theorem 1}

To obtain the finite singular points note that if $x=0$ then $y=0$, and if $y=0$ then $x=0$. Otherwise, if $x \neq 0$ and $y \neq 0$ we have

$$
y=x f(x, y) \quad \text { and } \quad x+x f^{2}(x, y)=x\left(1+f^{2}(x, y)\right)=0,
$$

which is never zero. In short, statement (a) is proved.

To prove statement (b) note that

$$
g(x, y)=x^{2}+y^{2}=0
$$

is an invariant algebraic curve whose cofactor is $2 f(x, y)$. By Proposition 7 we have that $\left(x^{2}+y^{2}\right)^{-(n+1) / 2}$ is an integrating factor of system (2). Consequently, in view of the Theorem 8 we conclude that system (2) has a Liouvillian first integral. This completes the proof of statement (b).

Statement (c) is an immediate consequence of Theorem 5.

Statement (d) is an immediate consequence of Theorem 6. Indeed, if system (2) has a limit cycle, since we have shown that it has an inverse integrating factor $\left(x^{2}+y^{2}\right)^{(n+1) / 2}$, in view of Theorem 6 the limit cycle must be contained in $x^{2}+y^{2}=0$, which is not possible. Hence, statement (d) is proved.

For statement (e) we compute the infinite singular points in the local chart $U_{1}$. Then, in the local chart $U_{1}$ system (2) becomes

$$
\dot{u}=-\left(1+u^{2}\right) v^{n-1}, \quad \dot{v}=-v\left(f(1, u)+u v^{n-1}\right) .
$$


Therefore all points $(u, 0)$ for all $u \in \mathbb{R}$ are infinite singular points in $U_{1}$. Note that the Jacobian matrix at $(u, 0)$ is of the form

$$
\left(\begin{array}{cc}
0 & 0 \\
0 & -f(1, u)
\end{array}\right)
$$

Hence the linear part of the singular points that are not special, that is, the points that after reparameterizating by the time $d s=v d t$ are not singular singular points of the system

$$
u^{\prime}=-\left(1+u^{2}\right) v^{n-2}, \quad v^{\prime}=-f(1, u)-u v^{n-1},
$$

is normally hyperbolic and so in view of the results in [10], on these singular points it starts or ends a unique orbit, implying that there are no global centers in system (2). This concludes the proof of statements (e) and (f).

From (5) it follows that in the local chart $U_{1}$ there are at most $n-1$ special singular points $(\bar{u}, 0)$. The special singular points are given by the real zeroes of $f(1, u)$ except if $f(x, y)=x^{n-1}$. If $f(x, y) \neq x^{n-1}$ and $n$ is even then $f(1, u)$ has odd degree and so it has at least one real zero, implying that there is at least one special singularity. Assume now that $f(x, y) \neq x^{n-1}, n$ is odd, the origin is a center and $f(1, u)$ has no real zeros. Then the polynomial $f(x, y)$ is written in the form $f(x, y)=\prod_{j=1}^{(n-1) / 2}\left(\alpha_{j} x^{2}+\beta_{j} x y+\gamma_{j} y^{2}\right)$ with $\beta_{j}^{2}-4 \alpha_{j} \gamma_{j}<0$ for $j=1, \ldots,(n-1) / 2$. Then clearly

$$
\left.\int_{0}^{2 \pi} f(x, y)\right|_{x=\cos \theta, y=\sin \theta} d \theta>0
$$

contradicting condition (3). In short, statement (g) is proved.

Now we start the proof of statement (h). Under our assumption all the singular points $\bar{u}$ of system (5) are simple zeros of $f(1, u)$, that is $f^{\prime}(1, \bar{u}) \neq 0$. Since the linear part of system (5) at the singular points is

$$
\left(\begin{array}{cc}
0 & 0 \\
-f^{\prime}(1, \bar{u}) & 0
\end{array}\right)
$$

the singular point $(\bar{u}, 0)$ is nilpotent. Since at most one more additional infinite singular point can appear, which is the origin of the local chart $U_{2}$, without loss of generality we can assume that all special infinite singular points of system (5) are on the local chart $U_{1}$, otherwise doing a rotation in the coordinates $(x, y)$ this would be the case. So in what follows we do not need to study whether the origin of the local chart $U_{2}$ is a special infinite singular point.

To study the special infinite singular points of system (5) (and so of system (2)) we write

$$
f(1, u)=\prod_{j=1}^{\ell_{0}}\left(u-r_{j}\right) \prod_{k=0}^{\ell_{1}}\left(u^{2}+\beta_{k} x+\gamma_{k}\right)
$$


where $\ell_{0}$ is a positive integer, $\ell_{1}$ is a non-negative integer and $r_{1}<r_{2}<\cdots<$ $r_{\ell_{0}}$. Now we study the singular point $\left(r_{p}, 0\right)$ with $1 \leq p \leq \ell_{0}$. We perform a translation to the origin of the point $\left(r_{p}, 0\right)$ taking the new variables

$$
U=u-r_{p}, \quad V=v .
$$

In these new variables system (5) becomes

(6)

$$
\begin{aligned}
U^{\prime}= & -\left(1+r_{p}^{2}+2 r_{p} U+U^{2}\right) V^{n-2} \\
V^{\prime}= & -U \prod_{\substack{j=1, j \neq p \\
\ell_{0}}}\left(U-\left(r_{j}-r_{p}\right)\right) \prod_{k=0}^{\ell_{1}}\left(U^{2}+\left(\beta_{k}+2 r_{p}\right) U+r_{p}^{2}+2 r_{p} \beta_{k}+\gamma_{k}\right) \\
& -\left(U+r_{p}\right) V^{n-1} .
\end{aligned}
$$

Note that in order to write this system under the normal form for applying Theorem 9 we make a scaling. Note that

$$
\begin{aligned}
& U^{\prime}=-\left(1+r_{p}^{2}\right) V^{n-2}-2 r_{p} U V^{n-2}-U^{2} V^{n-2}, \\
& V^{\prime}=A_{0} U+A_{1} U^{2}+\ldots+A_{\ell_{0}} U^{\ell_{0}}-r_{p} V^{n-1}-U V^{n-1},
\end{aligned}
$$

where

$$
A_{0}=(-1)^{\ell_{0}} \prod_{j=1, j \neq p}^{\ell_{0}}\left(r_{j}-r_{p}\right) \prod_{k=0}^{\ell_{1}}\left(r_{p}^{2}+2 r_{p} \beta_{k}+\gamma_{k}\right) \neq 0,
$$

and $A_{1}, A_{2}, \ldots, A_{\ell_{0}}$ belong to the higher order terms in $U$ for $V^{\prime}$. Now we introduce the scaling $d \tau=A_{0} d s$ and system (7) becomes

(8)

$$
\begin{aligned}
& \dot{U}=-\frac{1+r_{p}^{2}}{A_{0}} V^{n-2}+\frac{2 r_{p}}{A_{0}} U V^{n-2}+\frac{1}{A_{0}} U^{2} V^{n-2}=B(V, U), \\
& \dot{V}=U+\tilde{A}_{1} U^{2}+\ldots+\tilde{A}_{\ell_{0}} U^{\ell_{0}}-\frac{r_{p}}{A_{0}} V^{n-1}-\frac{1}{A_{0}} U V^{n-1}=U+A(V, U),
\end{aligned}
$$

where $\tilde{A}_{i}=A_{i} / A_{0}$ for $i=2, \ldots, \ell_{0}$ and the dot means derivative with respect to the new time $\tau$. Note that system (8) is in the normal form for applying Theorem 9 with $(x, y)=(V, U)$. Solving $U=f(V)$ we get that

$$
U=\frac{1+r_{p}^{2}}{A_{0}} V^{n-2}+\text { h.o.t. }
$$

Moreover

$$
F(V)=B(f(V), V)=-\frac{1+r_{p}^{2}}{A_{0}} V^{n-2}+\text { h.o.t. }
$$


and

$$
\begin{aligned}
G(V) & \left.=\left(\frac{\partial A}{\partial V}+\frac{\partial B}{\partial U}\right)(f(V), V)\right) \\
& =\frac{r_{p}(n-1)}{A_{0}} V^{n-2}+\text { h.o.t }+\frac{2 r_{p}}{A_{0}} V^{n-2}+\text { h.o.t } \\
& =\frac{r_{p}(n+1)}{A_{0}} V^{n-2}+\text { h.o.t. }
\end{aligned}
$$

If $n$ is even applying Theorem 9 we conclude that $\left(r_{p}, 0\right)$ is a cusp. Therefore modulo a translation to the origin and undoing the rescaling of time the local phase portrait for each singular point $\left(r_{p}, 0\right)$ might be one of the two shown in Figure 1. Moreover, recall that if $A_{0}$ is positive for $r_{p}$ then it is negative for $r_{p+1}$ and $r_{p-1}$ because $r_{p-1}<r_{p}<r_{p+1}$ and these zeroes are simple. Therefore between two cusps with local phase portrait as in Figure 1.A there must be a cusp with local phase portrait as in Figure 1.B. This concludes the proof of statement (h.1).

If $n$ is odd applying Theorem 9 we conclude that $\left(r_{p}, 0\right)$ is a saddle if $\left(1+r_{p}^{2}\right) / A_{0}<0$, and it is a focus or a center if $\left(1+r_{p}^{2}\right) / A_{0}>0$. Moreover, recall that if $A_{0}$ is positive for $r_{p}$ then it is negative for $r_{p+1}$ and $r_{p-1}$ because $r_{p-1}<r_{p}<r_{p+1}$. So, if $\left(r_{p}, 0\right)$ is a saddle, $\left(r_{p+1}, 0\right)$ and $\left(r_{p-1}, 0\right)$ must be foci or centers, or vice versa. Moreover, if $n$ is odd, $n-1$ is even and then $\ell_{0}$ is even. So there are $\ell_{0} / 2$ special singular points which are saddles and $\ell_{0} / 2$ special singular points which are foci or centers at the infinity in the local chart $U_{1}$. Moreover we have shown that they between two saddles there must be a center or a focus. This concludes the proof of statement (h.2) and concludes the proof of the theorem.

\section{PROOF OF THEOREM 3}

Note that system (2) is invariant under the change $(x, y) \mapsto(-x,-y)$. Therefore, this system is symmetric with respect to the origin and thus it is enough to study the phase portrait in the positive quadrant. Note that in this system there are either two special infinite singular points or four special singular points. If there are two special singular points then they are a saddle and a center or a focus. In this case the phase portrait is the same as the one in Figure 3. When there are four special singular points, then the local phase portrait is obtained in this case taking into account: the symmetry, the existence and uniqueness of the finite and infinite singular points; the existence and uniqueness theorem for the solutions of a differential system; the boundary of the Poincaré disc that consists entirely of singular points and the graphic at the boundary of the period annulus of the uniform isochronous center at the origin is formed by separatrices of the infinite singular points. Hence the global phase portraits in this case are topologically equivalent to the ones of Figures 5.A and 5.B. Moreover we have provided systems that 
realize these phase portraits in Figures 5.A and 5.B concluding the proof of the theorem.

\section{ACKNOWLEDGEMENTS}

The first author is supported by the Ministerio de Economía, Industria y Competitividad, Agencia Estatal de Investigación grant MTM201677278-P (FEDER), the Agència de Gestió d'Ajuts Universitaris i de Recerca grant 2017SGR1617, and the H2020 European Research Council grant MSCA-RISE-2017-777911. The second author is partially supported by FCT/Portugal through UID/MAT/04459/2013.

\section{REFERENCES}

[1] A. Algaba And M. Reyes, Characterizing isochronous points and computing isochronous sections, J. Math. Anal. Appl. 355 (2009), 564-576.

[2] J.C. ARTÉs, J. ItIKAWA AND J. LliBRe, Uniform isochronous cubic and quartic centers: Revisited, J. Comput. and Appl. Math. 313 (2017), 448-453.

[3] J. Chavarriga and M. Sabatini, A survey of isochronous centers, Qual. Theory Dynam. Syst. 1 (1999), 1-70.

[4] R. Conti, Uniformly isochronous centers of polynomial systems in $\mathbb{R}^{2}$, Lecture Notes in Pure and Appl. Math. 152 (1994), 21-31.

[5] F. Dumortier, J. Llibre and J. C. Artés Qualitative Theory of Planar Differential Systems, UniversiText, Springer-verlag, New York, 2006.

[6] G.R. Fowles And G.L. CAssiday, Analytic mechanics, Thomson Books/Cole, 2005.

[7] H. Giacomini, J. Llibre And M. Viano, On the nonexistence, existence and uniqueness of limit cycles, Nonlinearity 9 (1996), 501-516.

[8] A. G. Ghoudhury And P. GuHA, On commuting vector fields and Darboux functions for planar differential equations, Lobachevskii Journal of Mathematics 34 (2013), $212-226$.

[9] M. Han And V.G. Romanovski, Isochronicity and normal forms of polynomial systems of ODEs, J. Symb. Comput. 47 (2012), 1161-1174.

[10] M.H. Hirsch, C.C. Pugh AND M. Shub, Invariant manifolds, Lecture notes in mathematics 583, Springer-Verlag, New York 1977.

[11] J. ItikaWa And J. Llibre, Phase portraits of uniform isochronous centers, J. Comput. and Appl. Math. 287 (2015), 98-114.

[12] J. ITIKAWA AND J. LlibRe, Global phase portraits of uniform isochronous centers with quartic homogeneous polynomial nonlinearities, Discrete Cont. Dyn. Systems B 21 (2016), 121-131.

[13] W.S. Loud, Behavior of the period of solutions of certain plane autonomous systems near centers, Contributions to Diff. Eqs. 3 (1964), 21-36.

[14] V. RAmíREZ, Twin vector fields and independence of spectra for quadratic vector fields, J. Dyn. Control Syst. 23 (2017), 623-633.

[15] M.F. Singer, Liouvillian first integrals in differential equations Trans. Math. Soc. 333 (1992), 673-688.

\footnotetext{
${ }^{1}$ Departament de Matemàtiques, Universitat Autònoma de Barcelona, 08193 Bellaterra, Barcelona, Catalonia, Spain
}

Email address: jllibre@mat.uab.cat 
2 Departamento de Matemática, Instituto Superior Técnico, Universidade de Lisboa, Av. Rovisco Pais 1049-001, Lisboa, Portugal

Email address: cvalls@math.ist.utl.pt 